\title{
ON OPTIMIZATION OF MANUFACTURING OF A SENSE-AMPLIFIER BASED FLIP-FLOP MANUFACTURED IN A HETEROSTRUCTURES TO INCREASE DENSITY OF THEIR ELEMENTS. INFLUENCE OF MISMATCH INDUCED STRESS AND POROSITY OF MATERIALS ON TECHNOLOGICAL PROCESS
}

\section{E. L. PANKRATOV}

Nizhny Novgorod State University

23 Gagarin Avenue

Nizhny Novgorod 603950

Russia

Nizhny Novgorod State Technical University

24 Minin Street

Nizhny Novgorod 603950

Russia

e-mail: elp2004@mail.ru

\begin{abstract}
The paper describes an approach for increasing of density of field-effect heterotransistors in a sense-amplifier based flip-flop. To illustrate the approach we consider manufacturing of an amplifier of power in a heterostructure with specific configuration. One shall dope some specific areas of the heterostructure by diffusion or ion implantation. After that radiation defects and/or dopant
\end{abstract}

Keywords and phrases: broadband power amplifier, optimization of manufacturing, increasing of element integration rate.

Received October 12, 2019; Revised December 5, 2019

(C) 2020 Scientific Advances Publishers 
should be annealed framework optimized procedure. We introduce an approach for decreasing of stress between layers of heterostructure. Also it has been considered an analytical approach for prognosis of heat and mass transport in heterostructures, which can be take into account mismatch-induced stress.

\section{Introduction}

Currently some problems of electronic (to increase performance of solid state electronic devices, to increase their reliability, to increase density of integrated circuits elements: transistors, diodes) are solving [1-6]. Increasing of performance could be obtained by using materials, which have mobility of charge carriers with higher values [7-10]. Increasing of density of elements of integrated circuits could be obtained by optimized manufacturing of their elements in heterostructures with thin epitaxial layers [3-5,11-15]. In this situation dimensions of elements of integrated circuits could be decreased. Another approach to decrease these dimensions is using microwave annealing and laser annealing of radiation defects and/or dopants [16-18]. Our aim framework this paper is to formulate an approach to decrease dimensions of field-effect heterotransistors framework a broadband amplifier of power (see Figure 1) framework a heterostructure with specific configuration (epitaxial layer/porous buffer layer/substrate and sections in the epitaxial layer, manufactured by using another materials). 


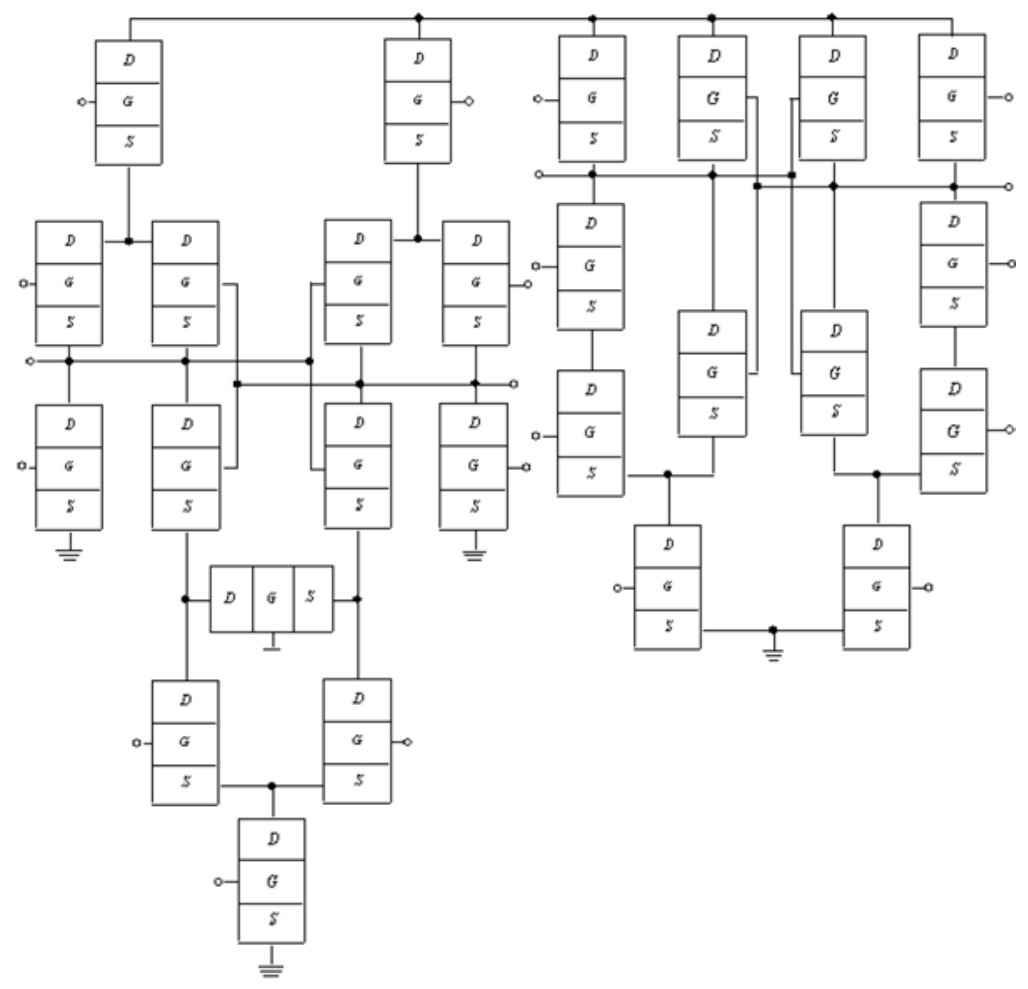

Figure 1(a). Scheme of the amplifier, which considered in this paper [19].

\begin{tabular}{|c|}
\hline Epitaxial layer \\
\hline Buffer layer \\
\hline Substrate \\
\\
\hline
\end{tabular}

Figure 1(b). Considered heterostructure, which include into itself epitaxial layers, buffer layer and substrate. 


\section{Method of Solution}

Now we will analyze distribution of concentration of dopant in space and time in heterostructure from Figure 1. To determine the concentration we solve the following second Fick's law [1, 20-24]:

$$
\begin{aligned}
\frac{\partial C(x, y, z, t)}{\partial t}= & \frac{\partial}{\partial x}\left[D \frac{\partial C(x, y, z, t)}{\partial x}\right]+\frac{\partial}{\partial y}\left[D \frac{\partial C(x, y, z, t)}{\partial y}\right]+\frac{\partial}{\partial z}\left[D \frac{\partial C(x, y, z, t)}{\partial z}\right] \\
& +\Omega \frac{\partial}{\partial x}\left[\frac{D_{S}}{k T} \nabla_{S} \mu_{1}(x, y, z, t) \int_{0}^{L_{z}} C(x, y, W, t) d W\right] \\
& +\Omega \frac{\partial}{\partial y}\left[\frac{D_{S}}{k T} \nabla_{S} \mu_{1}(x, y, z, t) \int_{0}^{L_{z}} C(x, y, W, t) d W\right] \\
& +\frac{\partial}{\partial x}\left[\frac{D_{C S}}{\bar{V} k T} \frac{\partial \mu_{2}(x, y, z, t)}{\partial x}\right]+\frac{\partial}{\partial y}\left[\frac{D_{C S}}{\bar{V} k T} \frac{\partial \mu_{2}(x, y, z, t)}{\partial y}\right] \\
& +\frac{\partial}{\partial z}\left[\frac{D_{C S}}{\bar{V} k T} \frac{\partial \mu_{2}(x, y, z, t)}{\partial z}\right] .
\end{aligned}
$$

Boundary and initial conditions for the equation could be written as

$$
\begin{array}{r}
\left.\frac{\partial C(x, y, z, t)}{\partial x}\right|_{x=0}=0,\left.\quad \frac{\partial C(x, y, z, t)}{\partial x}\right|_{x=L_{x}}=0,\left.\quad \frac{\partial C(x, y, z, t)}{\partial y}\right|_{y=0}=0 \\
C(x, y, z, 0)=f_{C}(x, y, z), \\
\left.\frac{\partial C(x, y, z, t)}{\partial y}\right|_{y=L_{y}}=0,\left.\quad \frac{\partial C(x, y, z, t)}{\partial z}\right|_{z=0}=0,\left.\quad \frac{\partial C(x, y, z, t)}{\partial z}\right|_{z=L_{z}}=0 .
\end{array}
$$

These boundary conditions correspond to absents of dopant flow through external boundary of heterostructure. The initial condition corresponds to distribution of dopant before starting of annealing (different for diffusive and ion types of annealing). Function $C(x, y, z, t)$ in Equations (1) and 
(1a) describes distribution of concentration of dopant in space and time; parameter $\Omega$ means volume of atom of dopant; symbol $\nabla_{s}$ means surficial gradient operator; function $\int_{0}^{L_{z}} C(x, y, z, t) d z$ describes dopant surficial concentration on interface between layers of heterostructure (we consider: $Z$-axis is perpendicular to interface between layers of heterostructure); functions $\mu_{1}(x, y, z, t)$ and $\mu_{2}(x, y, z, t)$ describe distributions of chemical potentials in space and their dependences on temperature: $\mu_{1}(x, y, z, t)$ accounting mismatch-induced stress in layers of heterostructure, $\mu_{2}(x, y, z, t)$ accounting porosity of material; functions $D(x, y, z, T)$ and $D_{S}(x, y, z, T)$ describe distributions of coefficients of volumetric and surficial diffusions in space and their dependences on temperature. We used recently described approximations of dopant diffusions coefficients [25-27]

$$
\begin{aligned}
& D_{C}=D_{L}(x, y, z, T)\left[1+\xi \frac{C^{\gamma}(x, y, z, t)}{P^{\gamma}(x, y, z, T)}\right]\left[1+\varsigma_{1} \frac{V(x, y, z, t)}{V^{*}}+\varsigma_{2} \frac{V^{2}(x, y, z, t)}{\left(V^{*}\right)^{2}}\right], \\
& D_{S}=D_{S L}(x, y, z, T)\left[1+\xi_{S} \frac{C^{\gamma}(x, y, z, t)}{P^{\gamma}(x, y, z, T)}\right]\left[1+\varsigma_{1} \frac{V(x, y, z, t)}{V^{*}}+\varsigma_{2} \frac{V^{2}(x, y, z, t)}{\left(V^{*}\right)^{2}}\right] .
\end{aligned}
$$

Functions $D_{L}(x, y, z, T)$ and $D_{L S}(x, y, z, T)$ describe dependences of dopant diffusion coefficients on coordinate and temperature; parameter $T$ describes temperature of annealing; function $P(x, y, z, T)$ describes dependence of solubility of dopant on coordinate and temperature; value of parameter $\gamma$ will be changed with changing of properties of materials and will be integer usually in the following interval $\gamma \in[1,3]$, [25]; function $V(x, y, z, t)$ describes distribution of radiation vacancies concentration in space and time with equilibrium distribution $V^{*}$. 
Distributions of point radiation defects concentration in space and time have been determined solution of the following system of equations [20-24, 26, 27]:

$$
\begin{aligned}
& \frac{\partial I(x, y, z, t)}{\partial t}=\frac{\partial}{\partial x}\left[D_{I}(x, y, z, T) \frac{\partial I(x, y, z, t)}{\partial x}\right] \\
& +\frac{\partial}{\partial y}\left[D_{I}(x, y, z, T) \frac{\partial I(x, y, z, t)}{\partial y}\right] \\
& +\frac{\partial}{\partial z}\left[D_{I}(x, y, z, T) \frac{\partial I(x, y, z, t)}{\partial z}\right] \\
& -k_{I, I}(x, y, z, T) I^{2}(x, y, z, t) \\
& -k_{I, V}(x, y, z, T) I(x, y, z, t) V(x, y, z, t) \\
& +\Omega \frac{\partial}{\partial x}\left[\frac{D_{I S}}{k T} \nabla_{S} \mu(x, y, z, t) \int_{0}^{L_{z}} I(x, y, W, t) d W\right] \\
& +\Omega \frac{\partial}{\partial y}\left[\frac{D_{I S}}{k T} \nabla_{S} \mu(x, y, z, t) \int_{0}^{L_{z}} I(x, y, W, t) d W\right] \\
& +\frac{\partial}{\partial x}\left[\frac{D_{I S}}{\bar{V} k T} \frac{\partial \mu_{2}(x, y, z, t)}{\partial x}\right] \\
& +\frac{\partial}{\partial y}\left[\frac{D_{I S}}{\bar{V} k T} \frac{\partial \mu_{2}(x, y, z, t)}{\partial y}\right]+\frac{\partial}{\partial z}\left[\frac{D_{I S}}{\bar{V} k T} \frac{\partial \mu_{2}(x, y, z, t)}{\partial z}\right] \\
& \frac{\partial V(x, y, z, t)}{\partial t}=\frac{\partial}{\partial x}\left[D_{V}(x, y, z, T) \frac{\partial V(x, y, z, t)}{\partial x}\right] \\
& +\frac{\partial}{\partial y}\left[D_{V}(x, y, z, T) \frac{\partial V(x, y, z, t)}{\partial y}\right] \\
& +\frac{\partial}{\partial z}\left[D_{V}(x, y, z, T) \frac{\partial V(x, y, z, t)}{\partial z}\right]
\end{aligned}
$$




$$
\begin{aligned}
& -k_{V, V}(x, y, z, T) V^{2}(x, y, z, t) \\
& -k_{I, V}(x, y, z, T) I(x, y, z, t) V(x, y, z, t) \\
& +\Omega \frac{\partial}{\partial x}\left[\frac{D_{V S}}{k T} \nabla_{S} \mu(x, y, z, t) \int_{0}^{L_{z}} V(x, y, W, t) d W\right] \\
& +\Omega \frac{\partial}{\partial y}\left[\frac{D_{V S}}{k T} \nabla_{S} \mu(x, y, z, t) \int_{0}^{L_{z}} V(x, y, W, t) d W\right] \\
& +\frac{\partial}{\partial x}\left[\frac{D_{V S}}{\bar{V} k T} \frac{\partial \mu_{2}(x, y, z, t)}{\partial x}\right] \\
& +\frac{\partial}{\partial y}\left[\frac{D_{V S}}{\bar{V} k T} \frac{\partial \mu_{2}(x, y, z, t)}{\partial y}\right]+\frac{\partial}{\partial z}\left[\frac{D_{V S}}{\bar{V} k T} \frac{\partial \mu_{2}(x, y, z, t)}{\partial z}\right] .
\end{aligned}
$$

Initial and boundary conditions for Equations (3) could be written as

$$
\begin{gathered}
\left.\frac{\partial I(x, y, z, t)}{\partial x}\right|_{x=0}=0,\left.\quad \frac{\partial I(x, y, z, t)}{\partial x}\right|_{x=L_{x}}=0,\left.\quad \frac{\partial I(x, y, z, t)}{\partial y}\right|_{y=0}=0, \\
\left.\frac{\partial I(x, y, z, t)}{\partial y}\right|_{y=L_{y}}=0,\left.\quad \frac{\partial I(x, y, z, t)}{\partial z}\right|_{z=0}=0,\left.\quad \frac{\partial I(x, y, z, t)}{\partial z}\right|_{z=L_{z}}=0, \\
\left.\frac{\partial V(x, y, z, t)}{\partial x}\right|_{x=0}=0,\left.\quad \frac{\partial V(x, y, z, t)}{\partial x}\right|_{x=L_{x}}=0,\left.\quad \frac{\partial V(x, y, z, t)}{\partial y}\right|_{y=0}=0, \\
\left.\frac{\partial V(x, y, z, t)}{\partial y}\right|_{y=L_{y}}=0,\left.\quad \frac{\partial V(x, y, z, t)}{\partial z}\right|_{z=0}=0,\left.\quad \frac{\partial V(x, y, z, t)}{\partial z}\right|_{z=L_{z}}=0, \\
I(x, y, z, 0)=f_{I}(x, y, z), V(x, y, z, 0)=f_{V}(x, y, z), \\
V\left(x_{1}+V_{n} t, y_{1}+V_{n} t, z_{1}+V_{n} t, t\right)=V_{\infty}\left(1+\frac{2 \ell \omega}{k T \sqrt{x_{1}^{2}+y_{1}^{2}+z_{1}^{2}}}\right) .
\end{gathered}
$$

Function $I(x, y, z, t)$ describes distribution of concentration of radiation interstitials (equilibrium distribution $I^{*}$ ) in space and time; functions 
$D_{I}(x, y, z, T), D_{V}(x, y, z, T), D_{I S}(x, y, z, T)$, and $D_{V S}(x, y, z, T)$ describe dependences of volumetric and surficial diffusions coefficients of vacancies and interstitials on coordinates and temperature; terms $I^{2}(x, y, z, t)$ and $V^{2}(x, y, z, t)$ describe generation diinterstitials and divacancies (see, for example, [27] and appropriate references in this book); functions $k_{I, V}(x, y, z, T), k_{I, I}(x, y, z, T)$, and $k_{V, V}(x, y, z, T)$ describe analogous dependences of parameters of recombination of point radiation defects and generation of their simplest complexes; parameter $k$ corresponds to Boltzmann constant; parameter $\omega$ is equal to $a^{3}$, where $a$ describes interatomic distance; parameter $\ell$ describes specific surface energy. Boundary conditions in Equations (4) correspond to absents of defects flow through external boundary of heterostructure. Initial conditions in Equations (4) correspond to distributions of defects before starting of annealing.

To take into account porosity of buffer layers we assume, that porous are approximately cylindrical with average values $r=\sqrt{x_{1}^{2}+y_{1}^{2}}$ and $z_{1}$ before annealing [24]. With time small pores will be decompose on vacancies. These vacancies will be absorbed by larger pores [28]. With time volume of large pores decreasing due to absorbing these vacancies and became more spherical [28]. Distribution of concentration of vacancies in heterostructure, existing due to porosity, could be determined by summing on all pores, i.e.,

$V(x, y, z, t)=\sum_{i=0}^{l} \sum_{j=0}^{m} \sum_{k=0}^{n} V_{P}(x+i \alpha, y+j \beta, z+k \chi, t), R=\sqrt{x^{2}+y^{2}+z^{2}}$.

Parameters $\alpha, \beta$, and $\chi$ describe average distances between centers of pores in directions $x, y$, and $z$, respectively; parameters $l, m$, and $n$ are the quantities of pores in appropriate directions. 
Distributions of diinterstitials $\Phi_{I}(x, y, z, t)$ and divacancies $\Phi_{V}(x, y, z, t)$ in space and time have been calculated as solutions of system of equations, which were presented below [26, 27]:

$$
\begin{aligned}
\frac{\partial \Phi_{I}(x, y, z, t)}{\partial t}= & \frac{\partial}{\partial x}\left[D_{\Phi_{I}}(x, y, z, T) \frac{\partial \Phi_{I}(x, y, z, t)}{\partial x}\right] \\
& +\frac{\partial}{\partial y}\left[D_{\Phi_{I}}(x, y, z, T) \frac{\partial \Phi_{I}(x, y, z, t)}{\partial y}\right] \\
& +\frac{\partial}{\partial z}\left[D_{\Phi_{I}}(x, y, z, T) \frac{\partial \Phi_{I}(x, y, z, t)}{\partial z}\right] \\
& +\Omega \frac{\partial}{\partial x}\left[\frac{D_{\Phi_{I} S}}{k T} \nabla_{S} \mu_{1}(x, y, z, t) \int_{0}^{L_{z}} \Phi_{I}(x, y, W, t) d W\right] \\
& +\Omega \frac{\partial}{\partial y}\left[\frac{D_{\Phi_{I}} S}{k T} \nabla_{S} \mu_{1}(x, y, z, t) \int_{0}^{L_{z}} \Phi_{I}(x, y, W, t) d W\right] \\
& +k_{I, I}(x, y, z, T) I^{2}(x, y, z, t) \\
& +\frac{\partial}{\partial x}\left[\frac{D_{\Phi_{I} S}}{\bar{V} k T} \frac{\partial \mu_{2}(x, y, z, t)}{\partial x}\right]+\frac{\partial}{\partial y}\left[\frac{D_{\Phi_{I} S}}{\bar{V} k T} \frac{\partial \mu_{2}(x, y, z, t)}{\partial y}\right] \\
& +\frac{\partial}{\partial z}\left[\frac{D_{\Phi_{I} S}}{\bar{V} k T} \frac{\partial \mu_{2}(x, y, z, t)}{\partial z}\right]+k_{I}(x, y, z, T) I(x, y, z, t) ;
\end{aligned}
$$

$$
\begin{aligned}
\frac{\partial \Phi_{V}(x, y, z, t)}{\partial t}= & \frac{\partial}{\partial x}\left[D_{\Phi_{V}}(x, y, z, T) \frac{\partial \Phi_{V}(x, y, z, t)}{\partial x}\right] \\
& +\frac{\partial}{\partial y}\left[D_{\Phi_{V}}(x, y, z, T) \frac{\partial \Phi_{V}(x, y, z, t)}{\partial y}\right] \\
& +\frac{\partial}{\partial z}\left[D_{\Phi_{V}}(x, y, z, T) \frac{\partial \Phi_{V}(x, y, z, t)}{\partial z}\right]
\end{aligned}
$$




$$
\begin{aligned}
& +\Omega \frac{\partial}{\partial x}\left[\frac{D_{\Phi_{V} S}}{k T} \nabla_{S} \mu_{1}(x, y, z, t) \int_{0}^{L_{z}} \Phi_{V}(x, y, W, t) d W\right] \\
& +\Omega \frac{\partial}{\partial y}\left[\frac{D_{\Phi_{V} S}}{k T} \nabla_{S} \mu_{1}(x, y, z, t) \int_{0}^{L_{z}} \Phi_{V}(x, y, W, t) d W\right] \\
& +k_{V, V}(x, y, z, T) V^{2}(x, y, z, t) \\
& +\frac{\partial}{\partial x}\left[\frac{D_{\Phi_{V} S}}{\bar{V} k T} \frac{\partial \mu_{2}(x, y, z, t)}{\partial x}\right]+\frac{\partial}{\partial y}\left[\frac{D_{\Phi_{V} S}}{\bar{V} k T} \frac{\partial \mu_{2}(x, y, z, t)}{\partial y}\right] \\
& +\frac{\partial}{\partial z}\left[\frac{D_{\Phi_{V} S}}{\bar{V} k T} \frac{\partial \mu_{2}(x, y, z, t)}{\partial z}\right]+k_{V}(x, y, z, T) V(x, y, z, t) .
\end{aligned}
$$

Initial and boundary conditions for above equations takes the form

$$
\begin{gathered}
\left.\frac{\partial \Phi_{I}(x, y, z, t)}{\partial x}\right|_{x=0}=0,\left.\frac{\partial \Phi_{I}(x, y, z, t)}{\partial x}\right|_{x=L_{x}}=0,\left.\frac{\partial \Phi_{I}(x, y, z, t)}{\partial y}\right|_{y=0}=0 \\
\left.\frac{\partial \Phi_{I}(x, y, z, t)}{\partial y}\right|_{y=L_{y}}=0,\left.\frac{\partial \Phi_{I}(x, y, z, t)}{\partial z}\right|_{z=0}=0,\left.\frac{\partial \Phi_{I}(x, y, z, t)}{\partial z}\right|_{z=L_{z}}=0 \\
\left.\frac{\partial \Phi_{V}(x, y, z, t)}{\partial x}\right|_{x=0}=0,\left.\frac{\partial \Phi_{V}(x, y, z, t)}{\partial x}\right|_{x=L_{x}}=0,\left.\frac{\partial \Phi_{V}(x, y, z, t)}{\partial y}\right|_{y=0}=0 \\
\left.\frac{\partial \Phi_{V}(x, y, z, t)}{\partial y}\right|_{y=L_{y}}=0,\left.\frac{\partial \Phi_{V}(x, y, z, t)}{\partial z}\right|_{z=0}=0,\left.\frac{\partial \Phi_{V}(x, y, z, t)}{\partial z}\right|_{z=L_{z}}=0 \\
\Phi_{I}(x, y, z, 0)=f_{\Phi I}(x, y, z), \quad \Phi_{V}(x, y, z, 0)=f_{\Phi V}(x, y, z) .
\end{gathered}
$$

Functions $D_{\Phi I S}(x, y, z, T), D_{\Phi V S}(x, y, z, T), D_{\Phi I}(x, y, z, T)$, and $D_{\Phi V}(x, y, z, T)$ describe dependences of surficial and volumetric diffusions of complexes of radiation defects on coordinate and temperature; functions $k_{I}(x, y, z, T)$ and $k_{V}(x, y, z, T)$ describe analogous dependences of parameters of decay of the above complexes. 
Boundary conditions in Equations (4) correspond to absents of defects flow through external boundary of heterostructure. Initial conditions in Equations (4) correspond to distributions of defects before starting of annealing. We determined chemical potential $\mu_{1}$ by the following relation [20]:

$$
\mu_{1}=E(z) \Omega \sigma_{i j}\left[u_{i j}(x, y, z, t)+u_{j i}(x, y, z, t)\right] / 2 .
$$

Function $E(z)$ describes spatial dependences of Young modulus; value $\sigma_{i j}$ describes tensor of stress; value $u_{i j}=\frac{1}{2}\left(\frac{\partial u_{i}}{\partial x_{j}}+\frac{\partial u_{j}}{\partial x_{i}}\right)$ describes tensor of deformation; values $u_{i}, u_{j}$ describe the displacement vector $\vec{u}(x, y, z, t) \quad$ with $\quad$ components $\quad u_{x}(x, y, z, t), u_{y}(x, y, z, t), \quad$ and $u_{z}(x, y, z, t) ; x_{i}, x_{j}$ are the coordinate $x, y, z$. The Equations (3) could be transform to the following form:

$$
\begin{aligned}
\mu(x, y, z, t)= & {\left[\frac{\partial u_{i}(x, y, z, t)}{\partial x_{j}}+\frac{\partial u_{j}(x, y, z, t)}{\partial x_{i}}\right]\left\{\frac { 1 } { 2 } \left[\frac{\partial u_{i}(x, y, z, t)}{\partial x_{j}}\right.\right.} \\
& \left.+\frac{\partial u_{j}(x, y, z, t)}{\partial x_{i}}\right]-\varepsilon_{0} \delta_{i j}+\frac{\sigma(z) \delta_{i j}}{1-2 \sigma(z)}\left[\frac{\partial u_{k}(x, y, z, t)}{\partial x_{k}}-3 \varepsilon_{0}\right] \\
& \left.-K(z) \beta(z)\left[T(x, y, z, t)-T_{0}\right] \delta_{i j}\right\} \frac{\Omega}{2} E(z) .
\end{aligned}
$$

Value $\sigma$ describes the value of coefficient of Poisson; value $\varepsilon_{0}=\left(a_{s}-a_{E L}\right) \quad / a_{E L}$ describes value of mismatch parameter, where parameters $a_{s}, a_{E L}$ describe value of lattice distances in the considered substrate and the epitaxial layer, respectively; parameter $K$ describes value of the uniform compression modulus; parameter $\beta$ describes value of thermal expansion coefficient; parameter $T_{r}$ describes value of equilibrium temperature, which we consider as room temperature. We 
consider components of displacement vector as solutions of the following equations [21]:

$$
\begin{aligned}
& \rho(z) \frac{\partial^{2} u_{x}(x, y, z, t)}{\partial t^{2}}=\frac{\partial \sigma_{x x}(x, y, z, t)}{\partial x}+\frac{\partial \sigma_{x y}(x, y, z, t)}{\partial y}+\frac{\partial \sigma_{x z}(x, y, z, t)}{\partial z}, \\
& \rho(z) \frac{\partial^{2} u_{y}(x, y, z, t)}{\partial t^{2}}=\frac{\partial \sigma_{y x}(x, y, z, t)}{\partial x}+\frac{\partial \sigma_{y y}(x, y, z, t)}{\partial y}+\frac{\partial \sigma_{y z}(x, y, z, t)}{\partial z}, \\
& \rho(z) \frac{\partial^{2} u_{z}(x, y, z, t)}{\partial t^{2}}=\frac{\partial \sigma_{z x}(x, y, z, t)}{\partial x}+\frac{\partial \sigma_{z y}(x, y, z, t)}{\partial y}+\frac{\partial \sigma_{z z}(x, y, z, t)}{\partial z} .
\end{aligned}
$$

Here $\quad \sigma_{i j}=\frac{E(z)}{2[1+\sigma(z)]}\left[\frac{\partial u_{i}(x, y, z, t)}{\partial x_{j}}+\frac{\partial u_{j}(x, y, z, t)}{\partial x_{i}}-\frac{\delta_{i j}}{3} \frac{\partial u_{k}(x, y, z, t)}{\partial x_{k}}\right]$

$+K(z) \delta_{i j} \frac{\partial u_{k}(x, y, z, t)}{\partial x_{k}}-\beta(z) K(z)\left[T(x, y, z, t)-T_{r}\right], \quad$ where function $\rho(z)$ describes density of materials of heterostructure and parameter $\delta_{i j}$ describes symbol of Kronecker. Accounting of the relation for $\sigma_{i j}$ in the last system of equation leads to the following relation:

$$
\begin{aligned}
\rho(z) \frac{\partial^{2} u_{x}(x, y, z, t)}{\partial t^{2}=} & \left\{K(z)+\frac{5 E(z)}{6[1+\sigma(z)]}\right\} \frac{\partial^{2} u_{x}(x, y, z, t)}{\partial x^{2}} \\
& +\left\{K(z)-\frac{E(z)}{3[1+\sigma(z)]}\right\} \frac{\partial^{2} u_{y}(x, y, z, t)}{\partial x \partial y} \\
& +\frac{E(z)}{2[1+\sigma(z)]}\left[\frac{\partial^{2} u_{y}(x, y, z, t)}{\partial y^{2}}+\frac{\partial^{2} u_{z}(x, y, z, t)}{\partial z^{2}}\right] \\
& +\left[K(z)+\frac{E(z)}{3[1+\sigma(z)]}\right] \frac{\partial^{2} u_{z}(x, y, z, t)}{\partial x \partial z} \\
& -K(z) \beta(z) \frac{\partial T(x, y, z, t)}{\partial x} ;
\end{aligned}
$$




$$
\begin{aligned}
& \rho(z) \frac{\partial^{2} u_{y}(x, y, z, t)}{\partial t^{2}}=\frac{E(z)}{2[1+\sigma(z)]}\left[\frac{\partial^{2} u_{y}(x, y, z, t)}{\partial x^{2}}+\frac{\partial^{2} u_{x}(x, y, z, t)}{\partial x \partial y}\right] \\
& -\frac{\partial T(x, y, z, t)}{\partial y} K(z) \beta(z) \\
& +\frac{\partial}{\partial z}\left\{\frac{E(z)}{2[1+\sigma(z)]}\left[\frac{\partial u_{y}(x, y, z, t)}{\partial z}+\frac{\partial u_{z}(x, y, z, t)}{\partial y}\right]\right\} \\
& +\frac{\partial^{2} u_{y}(x, y, z, t)}{\partial y^{2}}\left\{\frac{5 E(z)}{12[1+\sigma(z)]}+K(z)\right\} \\
& +\left\{K(z)-\frac{E(z)}{6[1+\sigma(z)]}\right\} \frac{\partial^{2} u_{y}(x, y, z, t)}{\partial y \partial z} \\
& +K(z) \frac{\partial^{2} u_{y}(x, y, z, t)}{\partial x \partial y} \\
& \rho(z) \frac{\partial^{2} u_{z}(x, y, z, t)}{\partial t^{2}}=\frac{E(z)}{2[1+\sigma(z)]}\left[\frac{\partial^{2} u_{z}(x, y, z, t)}{\partial x^{2}}+\frac{\partial^{2} u_{z}(x, y, z, t)}{\partial y^{2}}\right. \\
& \left.+\frac{\partial^{2} u_{x}(x, y, z, t)}{\partial x \partial z}+\frac{\partial^{2} u_{y}(x, y, z, t)}{\partial y \partial z}\right] \\
& +\frac{\partial}{\partial z}\left\{K(z)\left[\frac{\partial u_{x}(x, y, z, t)}{\partial x}+\frac{\partial u_{y}(x, y, z, t)}{\partial y}+\frac{\partial u_{x}(x, y, z, t)}{\partial z}\right]\right\} \\
& +\frac{1}{6} \frac{\partial}{\partial z}\left\{\frac { E ( z ) } { 1 + \sigma ( z ) } \left[6 \frac{\partial u_{z}(x, y, z, t)}{\partial z}-\frac{\partial u_{x}(x, y, z, t)}{\partial x}\right.\right. \\
& \left.\left.-\frac{\partial u_{y}(x, y, z, t)}{\partial y}-\frac{\partial u_{z}(x, y, z, t)}{\partial z}\right]\right\} \\
& -K(z) \beta(z) \frac{\partial T(x, y, z, t)}{\partial z} .
\end{aligned}
$$


Conditions for the above equations takes the form

$\frac{\partial \vec{u}(0, y, z, t)}{\partial x}=0 ; \frac{\partial \vec{u}\left(L_{x}, y, z, t\right)}{\partial x}=0 ; \frac{\partial \vec{u}(x, 0, z, t)}{\partial y}=0 ; \frac{\partial \vec{u}\left(x, L_{y}, z, t\right)}{\partial y}=0 ;$

$\frac{\partial \vec{u}(x, y, 0, t)}{\partial z}=0 ; \frac{\partial \vec{u}\left(x, y, L_{z}, t\right)}{\partial z}=0 ; \vec{u}(x, y, z, 0)=\vec{u}_{0} ; \vec{u}(x, y, z, \infty)=\vec{u}_{0}$.

We calculate solutions of Equations (1), (3), (5), and (8) by using standard method of averaging of function corrections [29]. We will not present the solutions, because we presented analogous solution in our present works (see [12] and our similar works).

\section{Discussion}

Now we will analyzing redistributions of radiation defects and dopant during annealing. In this situation, we take into account influence of modification of porosity and mismatch-induced stress on redistribution of radiation defects and dopant. We present several spatial distributions of concentrations of dopant in heterostructures on Figures 2 and 3. Figure 2 corresponds to diffusion type of doping. Figure 3 corresponds to ion type of doping. All distributions of dopant correspond to larger value of dopant diffusion coefficient in comparison with nearest areas. One can find that inhomogeneity of heterostructure leads to increasing homogeneity of dopant in doped area of epitaxial layer. 


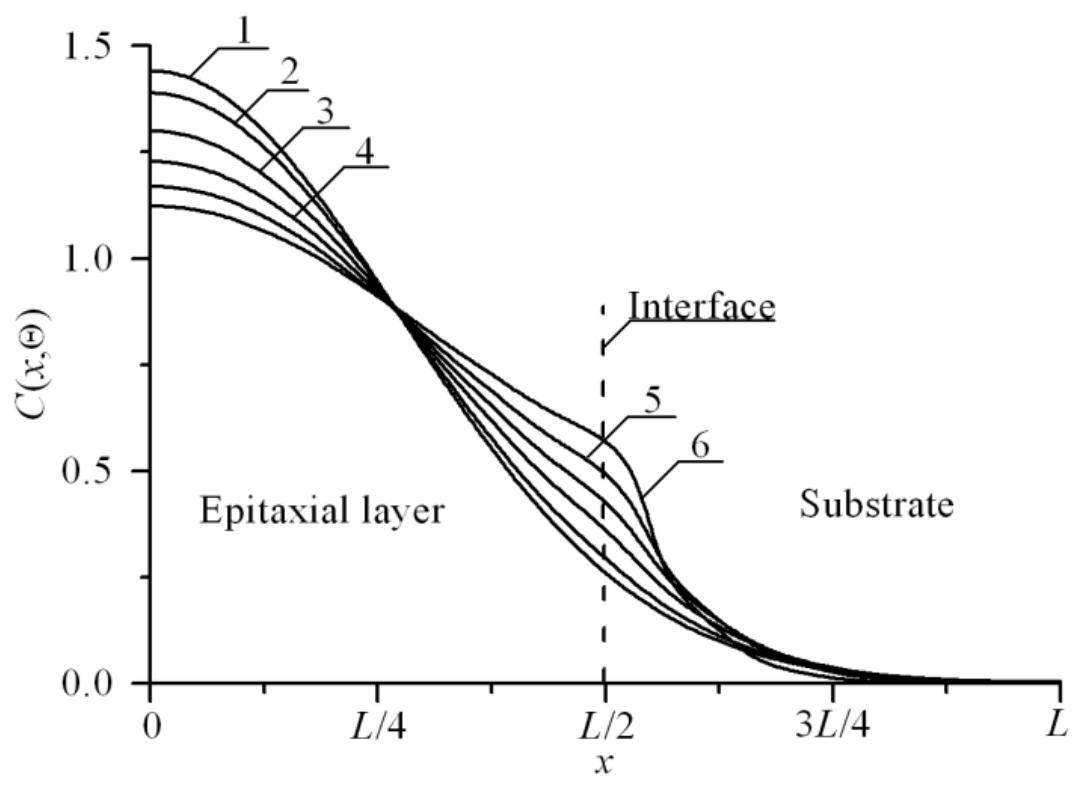

Figure 2. Spatial distributions of infused dopant concentration in the considered heterostructure in perpendicular direction to interface between epitaxial layer and substrate. Larger number of curves corresponds to larger difference between values of dopant diffusion coefficient in the heterostructure. Value of dopant diffusion coefficient in the substrate is smaller, than value of dopant diffusion coefficient in the epitaxial layer. 


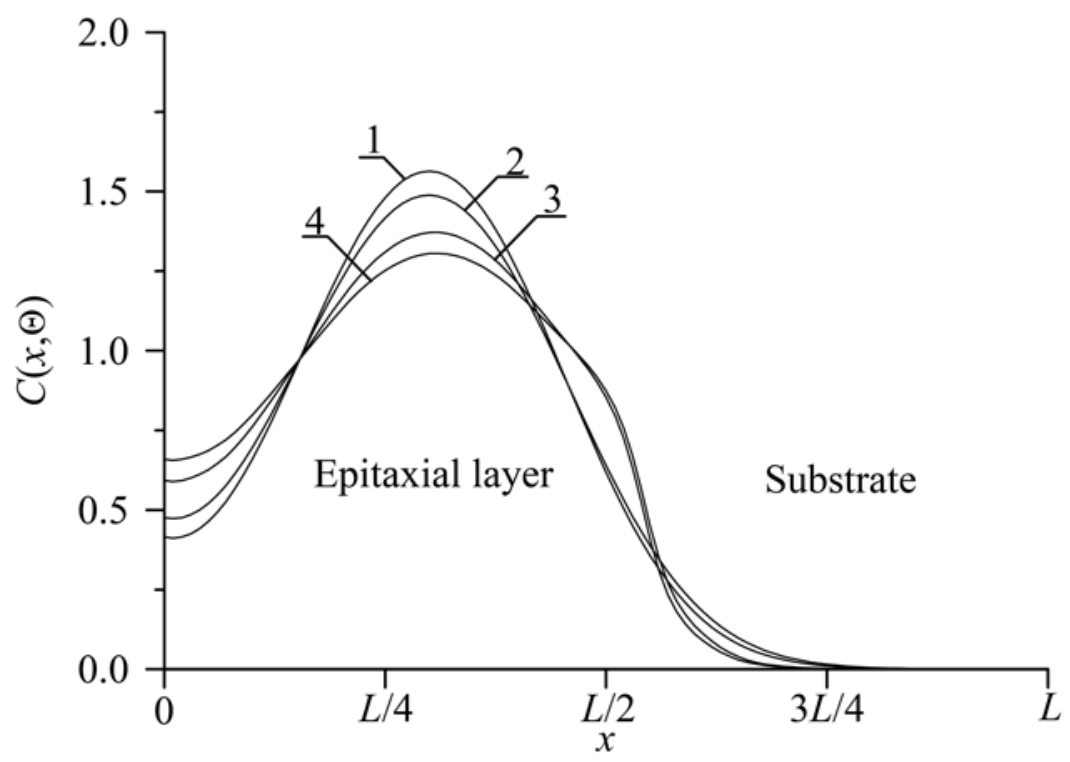

Figure 3. Spatial distributions of implanted dopant concentration in the considered heterostructure in perpendicular direction to interface between epitaxial layer and substrate. Curves 1 and 3 were calculated for time of annealing $\Theta=0.0048\left({L_{x}}^{2}+{L_{y}}^{2}+{L_{z}}^{2}\right) / D_{0}$. Curves 2 and 4 were calculated for time of annealing $\Theta=0.0057\left(L_{x}{ }^{2}+L_{y}{ }^{2}+L_{z}{ }^{2}\right) / D_{0}$. Curves 1 and 2 were calculated for homogeneous sample. Curves 3 and 4 were calculated for heterostructure for the case, when diffusion coefficient of dopant in the substrate is smaller, than diffusion coefficient of dopant in epitaxial layer.

However, it should be noted that optimization of annealing of dopant and/or radiation defects required to obtain compromise between increasing of homogeneity of distribution of concentration of dopant in required area and increasing of sharpness of this distribution after this area. To obtain this compromise we used recently introduced criterion [12, 30-37]. To use this criterion one shall approximate real distribution of concentration of dopant by step-wise function (see Figures 4 and 5). 
Farther we determine optimal values of annealing time by minimization of the following mean-squared error:

$$
U=\frac{1}{L_{x} L_{y} L_{z}} \int_{0}^{L_{x}} \int_{0}^{L_{y}} \int_{0}^{L_{z}}[C(x, y, z, \Theta)-\psi(x, y, z)] d z d y d x
$$

to determine compromise value of annealing time. Here $\psi(x, y, z)$ is the considered step-wise approximation function.

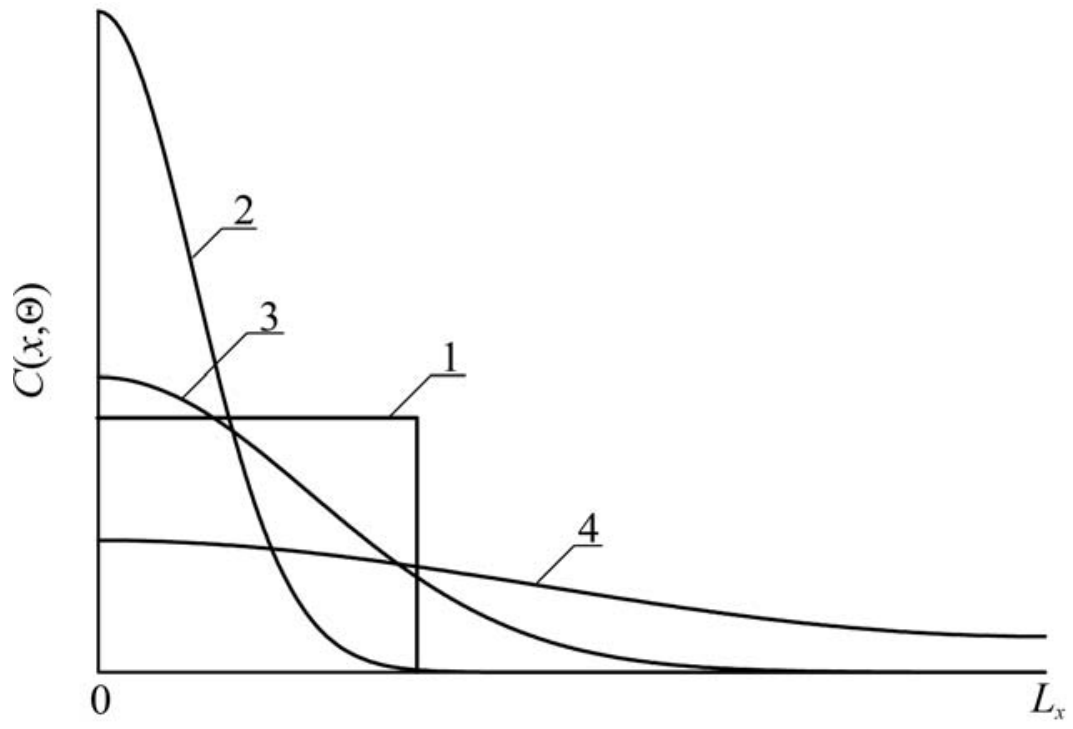

Figure 4. Spatial distributions of concentration of the infused dopant in the considered heterostructure. Curve 1 shows idealized dopant distribution (step-wise function). Curves 2-4 show real dopant distributions at different continuance of annealing. Larger value of number of curve mean larger value of continuance of annealing. 


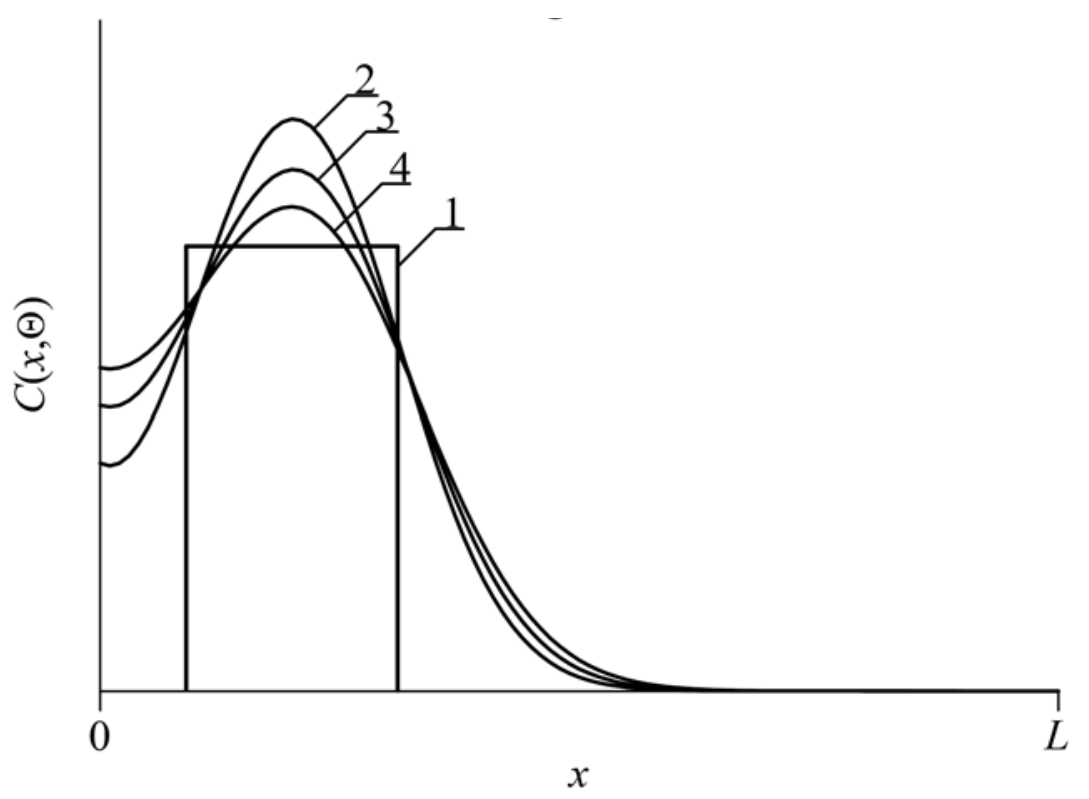

Figure 5. Spatial distributions of concentration of the implanted dopant in the considered heterostructure. Curve 1 shows idealized dopant distribution (step-wise function). Curves 2-4 show real dopant distributions at different continuance of annealing.

Figure 6 (for diffusion type of doping) and Figure 7 (for ion type of doping) show optimal annealing times as functions of several parameters. It is known, that anneal radiation defects after ion implantation should be done. Spatial distributions of dopant will spread during this annealing. The dopant should achieve required interface between layers of heterostructure during the annealing. If dopant can not achieve during the radiation defects annealing, it is attracted an interest additional annealing of the dopant. In this situation one can find smaller value of implanted dopant annealing time in comparison with annealing time of infused dopant. 


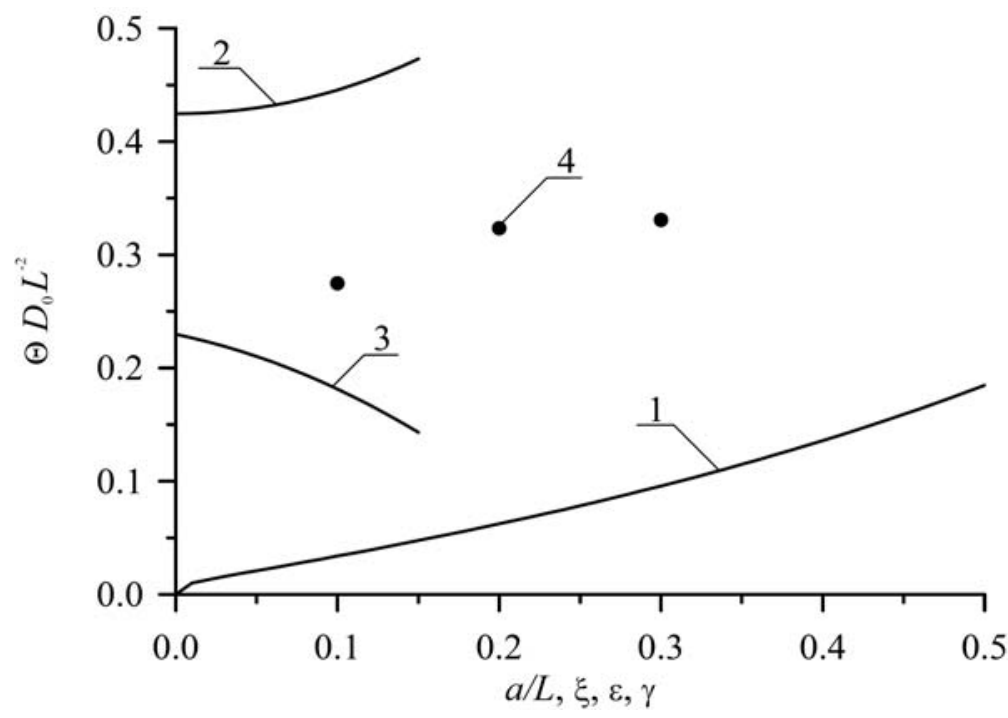

Figure 6. Dimensionless optimal annealing time of infused dopant as functions of several parameters. Curve 1 describes dependence of the annealing time on $a / L$ at $\xi=\gamma=0$ in homogeneous material. Curve 2 describes dependence of the annealing time on $\varepsilon$ at $a / L=1 / 2$ and $\xi=\gamma=0$. Curve 3 describes dependence of the annealing time on $\xi$ at $a / L=1 / 2$ and $\varepsilon=\gamma=0$. Curve 4 describes dependence of the annealing time on $\gamma$ at $a / L=1 / 2$ and $\varepsilon=\xi=0$. 


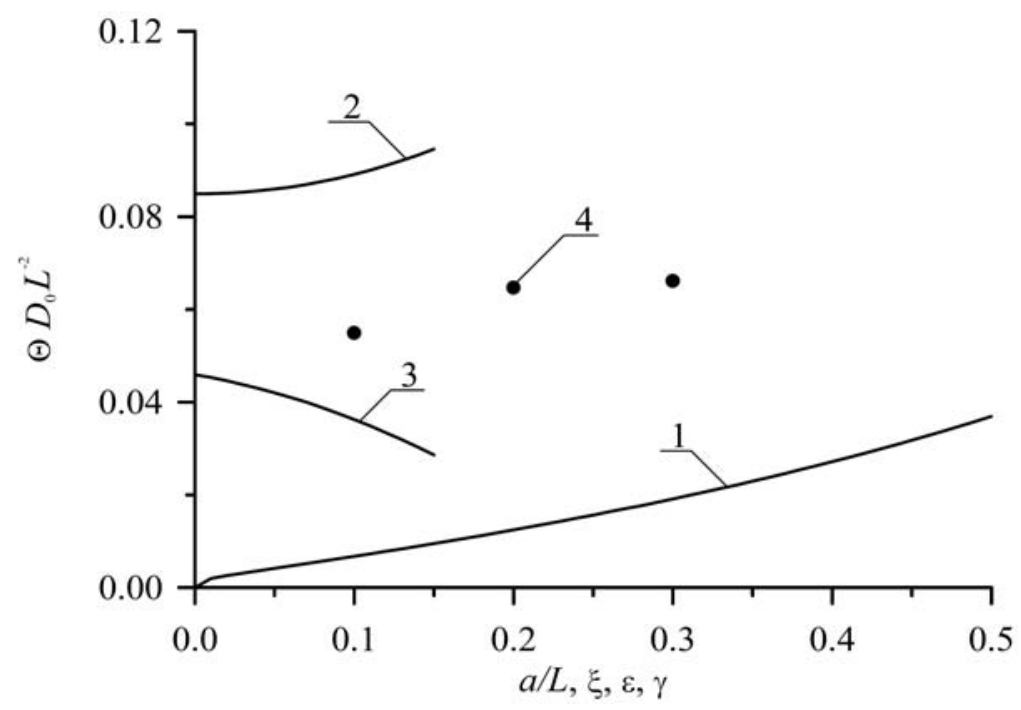

Figure 7. Dimensionless optimal annealing time of infused dopant as functions of several parameters. Curve 1 describes dependence of the annealing time on $a / L$ at $\xi=\gamma=0$ in homogeneous material. Curve 2 describes dependence of the annealing time on $\varepsilon$ at $a / L=1 / 2$ and $\xi=\gamma=0$. Curve 3 describes dependence of the annealing time on $\xi$ at $a / L=1 / 2$ and $\varepsilon=\gamma=0$. Curve 4 describes dependence of the annealing time on $\gamma$ at $a / L=1 / 2$ and $\varepsilon=\xi=0$.

Now we consider influence of variation of mismatch induced stress on dopant concentration distribution in the considered heterostructure. At $\varepsilon_{0}<0$ a compression of dopant concentration distribution could be find near interface between layers of heterostructure. On the other hand (at $\varepsilon_{0}>0$ ) spreading of the distribution could be find. The variation of distribution could be partially decreased by laser annealing [37] due to acceleration of dopant diffusion during this procedure. Presents of mismatch-induced stress in heterostructure leads to changing of optimal values of annealing time. At the same time modification of porosity leads to decrease value of mechanical stress. On the one hand mismatch- 
induced stress could be used to increase density of elements of integrated circuits. At the same time the stress could leads to generation dislocations of the discrepancy. Figures 8 and 9 show vacancies concentration distributions in porous materials and component of displacement vector, which is perpendicular to interface between layers of heterostructure.

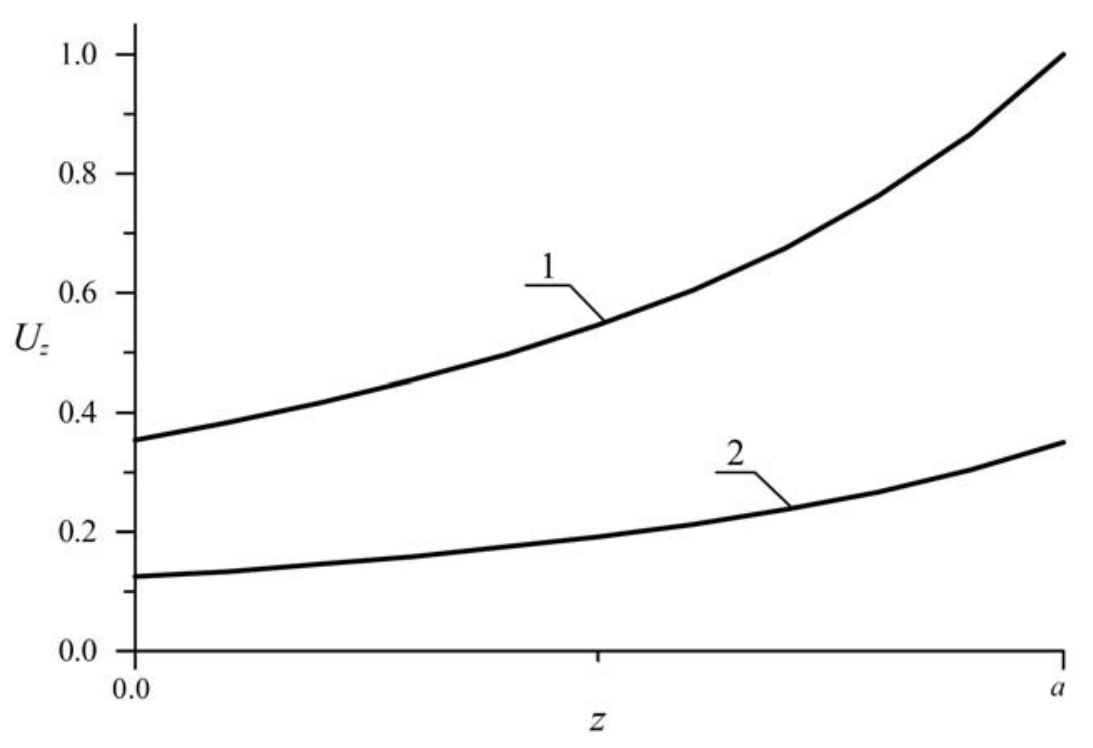

Figure 8. Dependences of normalized component of displacement vector on coordinate, which is perpendicular to interface between layers of heterostructure. Curve 1 corresponds to nonporous material. Curve 2 corresponds to porous material. 


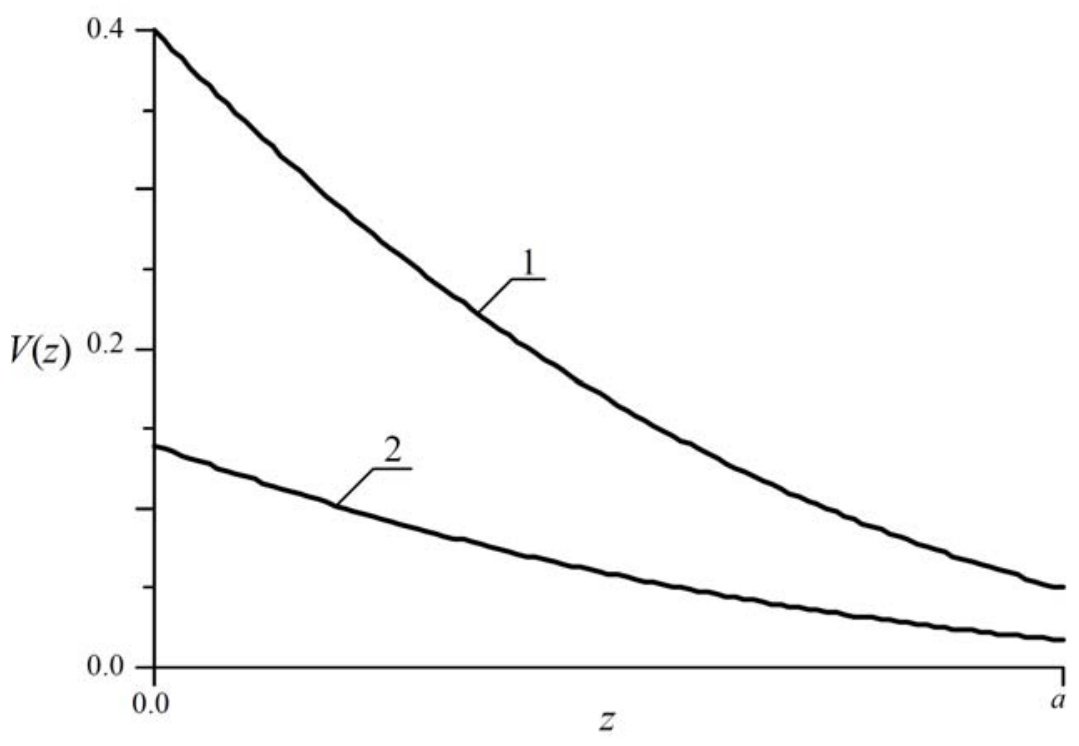

Figure 9. Dependences of normalized vacancy concentrations on coordinate, which is perpendicular to interface between layers of heterostructure. Curve 1 corresponds to nonporous material. Curve 2 corresponds to porous material.

\section{Conclusion}

We analyzed redistribution of implanted and infused dopants in a heterostructure with porous layer to manufacturing field-effect heterotransistors framework in a sense-amplifier based flip-flop. We also analyzed influence of relaxation mismatch-induced stress on above redistribution. We obtain several recommendations to optimize annealing for decreasing of dimensions of transistors and to increase their density framework an integrated circuits. We also formulate conditions for decreasing mismatch-induced stress. An analytical approach to solve considered boundary problems with account nonlinearity of processes and changing of parameters in space and time has been introduced. 


\section{References}

[1] V. I. Lachin and N. S. Savelov, Electronics, Rostov-on-Don, Phoenix, 2001.

[2] A. Polishscuk, Sovremennaya elektronika, Modern Electronics 12 (2004), 8-11 (in Russian).

[3] G. Volovich, Modern chips UM3Ch class D manufactured by firm MPS, Modern Electronics 2 (2006), 10-17.

[4] A. Kerentsev and V. Lanin, Constructive-technological features of MOSFETtransistors, Power Electronics 1 (2008), 34-38.

[5] A. O. Ageev, A. E. Belyaev, N. S. Boltovets, V. N. Ivanov, R. V. Konakova, Ya. Ya. Kudrik, P. M. Litvin, V. V. Milenin and A. V. Sachenko, Au-TiB $x-n-6 H-S i C$

Schottky barrier diodes: Specific features of charge transport in rectifying and nonrectifying contacts, Semiconductors 43(7) (2009), 865-871.

DOI: https://doi.org/10.1134/S1063782609070070

[6] Jung-Hui Tsai, Shao-Yen Chiu, Wen-Shiung Lour and Der-Feng Guo, Highperformance InGaP/GaAs pnp $\delta$-doped heterojunction bipolar transistor, Semiconductors 43(7) (2009), 939-942.

DOI: https://doi.org/10.1134/S1063782609070227

[7] O. V. Alexandrov, A. O. Zakhar'in, N. A. Sobolev, E. I. Shek, M. M. Makoviychuk and E. O. Parshin, Formation of donor centers upon annealing of dysprosium-and holmium-implanted silicon, Semiconductors 32(9) (1998), 921-923.

DOI: https://doi.org/10.1134/1.1187515

[8] I. B. Ermolovich, V. V. Milenin, R. A. Red'ko and S. M. Red'ko, Specific features of recombination processes in CdTe films produced in different temperature conditions of growth and subsequent annealing, Semiconductors 43(8) (2009), 980-984.

DOI: https://doi.org/10.1134/S106378260908003X

[9] P. Sinsermsuksakul, K. Hartman, S. B. Kim, J. Heo, L. Sun, H. H. Park, R. Chakraborty, T. Buonassisi and R. G. Gordon, Enhancing the efficiency of SnS solar cells via band-offset engineering with a zinc oxysulfide buffer layer, Applied Physics Letters 102(5) (2013); Article 053901.

DOI: https://doi.org/10.1063/1.4789855

[10] J. G. Reynolds, C. L. Reynolds, Jr. A. Mohanta, J. F. Muth, J. E. Rowe, H. O. Everitt and D. E. Aspnes, Shallow acceptor complexes in p-type ZnO, Applied Physics Letters 102(15) (2013); Article 152114.

DOI: https://doi.org/10.1063/1.4802753 
[11] N. I. Volokobinskaya, I. N. Komarov, T. V. Matyukhina, V. I. Reshetnikov, A. A. Rush, I. V. Falina and A. S. Yastrebov, A study of technological processes in the production of high-power high-voltage bipolar transistors incorporating an array of inclusions in the collector region, Semiconductors 35(8) (2001), 974-978.

DOI: https://doi.org/10.1134/1.1393038

[12] E. L. Pankratov and E. A. Bulaeva, Doping of materials during manufacture $p-n$-junctions and bipolar transistors: Analytical approaches to model technological approaches and ways of optimization of distributions of Dopants, Reviews in Theoretical Science 1(1) (2013), 58-82.

DOI: https://doi.org/10.1166/rits.2013.1004

[13] S. A. Kukushkin, A. V. Osipov and A. I. Romanychev, Epitaxial growth of zinc oxide by the method of atomic layer deposition on SiC/Si substrates, Physics of the Solid State 58(7) (2016), 1448-1452.

$$
\text { DOI: https://doi.org/10.1134/S1063783416070246 }
$$

[14] E. M. Trukhanov, A. V. Kolesnikov and I. D. Loshkarev, Long-range stresses generated by misfit dislocations in epitaxial films, Russian Microelectronics 44(8) (2015), 552-558.

DOI: https://doi.org/10.1134/S1063739715080119

[15] E. L. Pankratov and E. A. Bulaeva, On optimization of regimes of epitaxy from gas phase: Some analytical approaches to model physical processes in reactors for epitaxy from gas phase during growth films, Reviews in Theoretical Science 3(4) (2015), 365-398.

DOI: https://doi.org/10.1166/rits.2015.1041

[16] K. K. Ong, K. L. Pey, P. S. Lee, A. T. S. Wee, X. C. Wang and Y. F. Chong, Dopant distribution in the recrystallization transient at the maximum melt depth induced by laser annealing, Applied Physics Letters 89(17) (2006); Article 172111.

DOI: https://doi.org/10.1063/1.2364834

[17] H. T. Wang, L. S. Tan and E. F. Chor, Pulsed laser annealing of Be-implanted GaN, Journal of Applied Physics 98(9) (2006); Article 094901.

DOI: https://doi.org/10.1063/1.2120893

[18] Yu. V. Bykov, A. G. Yeremeev, N. A. Zharova, I. V. Plotnikov, K. I. Rybakov, M. N. Drozdov, Yu. N. Drozdov and V. D. Skupov, Diffusion processes in semiconductor structures during microwave annealing, Radiophysics and Quantum Electronics 46(8-9) (2003), 749-755.

DOI: https://doi.org/10.1023/B:RAQE.0000025008.97954.1c 
[19] Bo Chen, Li Shen, S. Liu, Y. Zheng and J. Gao, A broadband, high isolation millimeter-wave CMOS power amplifier using a transformer and transmission line matching topology, Analog Integrated Circuits and Signal Processing 81(2) (2014), 537-547.

$$
\text { DOI: https://doi.org/10.1007/s10470-014-0412-z }
$$

[20] Y. W. Zhang and A. F. Bower, Numerical simulations of island formation in a coherent strained epitaxial thin film system, Journal of the Mechanics and Physics of Solids 47(11) (1999), 2273-2297.

DOI: https://doi.org/10.1016/S0022-5096(99)00026-5

[21] L. D. Landau and E. M. Lefshits, Theoretical Physics 7 (Theory of Elasticity), Physmatlit, Moscow, 2001.

[22] M. Kitayama, T. Narushima, W. C. Carter, R. M. Cannon and A. M. Glaeser, The wulff shape of alumina: I, Modeling the kinetics of morphological evolution, Journal of the American Ceramic Society 83(10) (2000), 2561-2531.

DOI: https://doi.org/10.1111/j.1151-2916.2000.tb01591.x

[23] M. Kitayama, T. Narushima, The wulff shape of alumina: II, Experimental measurements of pore shape evolution rates, Journal of the American Ceramic Society 83(10) (2000), 2572-2583.

DOI: https://doi.org/10.1111/j.1151-2916.2000.tb01592.x

[24] P. G. Cheremskoy, V. V. Slesov and V. I. Betekhtin, Pore in Solid Bodies, Energoatomizdat, Moscow, 1990.

[25] Z. Yu. Gotra, Technology of Microelectronic Devices, Radio and Communication, Moscow, 1991.

[26] P. M. Fahey, P. B. Griffin and J. D. Plummer, Point defects and dopant diffusion in silicon, Reviews of Modern Physics 61(2) (1989), 289.

DOI: https://doi.org/10.1103/RevModPhys.61.289

[27] V. L. Vinetskiy and G. A. Kholodar, Radiative Physics of Semiconductors, Naukova Dumka, Kiev, 1979.

[28] M. G. Mynbaeva, E. N. Mokhov, A. A. Lavrent'ev and K. D. Mynbaev, Hightemperature diffusion doping of porous silicon carbide, Technical Physics Letters 34 (2008); Article 731.

DOI: https://doi.org/10.1134/S1063785008090034

[29] Yu. D. Sokolov, About the definition of dynamic forces in the mine lifting, Applied Mechanics 1(1) (1955), 23-35.

[30] E. L. Pankratov, Dopant diffusion dynamics and optimal diffusion time as influenced by diffusion-coefficient nonuniformity, Russian Microelectronics 36(1) (2007), 33-39.

DOI: https://doi.org/10.1134/S1063739707010040 
[31] E. L. Pankratov, Redistribution of a dopant during annealing of radiation defects in a multilayer structure by laser scans for production of an implanted-junction rectifier, International Journal of Nanoscience 7(4-5) (2008), 187-197.

DOI: https://doi.org/10.1142/S0219581X08005328

[32] E. L. Pankratov and E. A. Bulaeva, Decreasing of quantity of radiation defects in an implanted-junction rectifiers by using overlayers, International Journal of MicroNano Scale Transport 3(3) (2012), 119-130.

[33] E. L. Pankratov and E. A. Bulaeva, Optimization of manufacturing of emittercoupled logic to decrease surface of chip, International Journal of Modern Physics B 29(5) (2015); Article 1550023.

DOI: https://doi.org/10.1142/S021797921550023X

[34] E. L. Pankratov, On approach to optimize manufacturing of bipolar heterotransistors framework circuit of an operational amplifier to increase their integration rate: Influence mismatch-induced stress, Journal of Computational and Theoretical Nanoscience 14(10) (2017), 4885-4899.

DOI: https://doi.org/10.1166/jctn.2017.6899

[35] E. L. Pankratov and E. A. Bulaeva, An approach to increase the integration rate of planar drift heterobipolar transistors, Materials Science in Semiconductor Processing 34 (2015), 260-268.

DOI: https://doi.org/10.1016/j.mssp.2015.02.054

[36] E. L. Pankratov and E. A. Bulaeva, An approach to manufacture a heterobipolar transistors in thin film structures: On the method of optimization, International Journal of Micro-Nano Scale Transport 4(1) (2014), 17-31.

[37] E. L. Pankratov, Increasing of the sharpness of $p-n$ junctions by laser pulses, Nano 6(1) (2011), 31-40.

DOI: https://doi.org/10.1142/S1793292011002329 